\title{
Self-assessment of final-year undergraduate physiotherapy students' literature-searching behaviour, self-perceived knowledge of their own critical appraisal skills and evidence-based practice beliefs
}

D A Hess, MSc (Physiotherapy); J Frantz, PhD (Physiotherapy)

Department of Physiotherapy, Faculty of Community and Health Sciences, University of the Western Cape, Cape Town, South Africa

Corresponding author: D A Hess (hess.danelle@gmail.com)

Background. Health professionals and new graduates alike are expected to be efficient in applying evidence-based practice. Research and evidencebased practice skills are taught in the research modules at university, but it is not clear whether students translate those skills into clinical practice. Objective. To determine the self-assessment literature-searching behaviour, self-perceived knowledge of critical appraisal skills and evidence-based practice beliefs of final-year undergraduate physiotherapy students at a university in South Africa.

Methods. This study used a quantitative approach, with a small qualitative component. A convenience sample of the final-year undergraduate physiotherapy students $(N=36)$ registered for the 2012 academic year was approached to participate in the study. Data were collected using an existing questionnaire.

Results. The study yielded a $75 \%$ response rate. With regard to literature-searching skills, $30 \%$ searched for literature more than once a week, $52 \%$ searched only for specific information and most had access to literature. Students received their information from journal articles (85\%), the internet and databases. They were slightly confident when it came to literature appraisal and believed that evidence-based practice was essential to their practice (96\%), but expressed a need for more training (59\%). Their understanding of the evidence-based practice concept was limited because they based their treatment choices on content and other role-models, and related their choices to their previous patient experiences.

Conclusion. Students believed that evidence-based practice was vital, yet their understanding of the concept was restricted when compared with the literature and they expressed a need for further training.

Afr J Health Professions Educ 2016;8(2):174-177. DOI:10.7196/AJHPE.2016.v8i2.580

Evidence-based medicine is defined as 'the conscientious, explicit, and judicious use of current best evidence in making decisions about the care of individual patients..$^{[1]}$ An initial focus on medicine later expanded to include all health professional disciplines, with evidence-based medicine becoming evidence-based practice. ${ }^{[2]}$

As evidence-based practice emerged and grew, healthcare professionals and new graduates were expected to use and be confident in applying evidencebased practice skills. ${ }^{[3]}$ The Sicily Statement on evidence-based practice states that on graduation, healthcare professionals must have the skills to search for and appraise new knowledge and apply it to their practice, as well as begin an appreciation for life-long learning, to be able to adapt to changes throughout their careers. ${ }^{[4]}$ According to the Sicily Statement, the evidence-based practice process consists of five steps: (i) translation of uncertainty to an answerable question; (ii) systematic retrieval of best evidence available; (iii) critical appraisal of evidence for validity, clinical relevance and applicability; (iv) application of results in practice; and $(v)$ evaluation of performance. ${ }^{[4]}$ To perform these steps accurately requires skill, especially in the retrieval of appropriate evidence and critical appraisal. In the physiotherapy course currently offered by the University of the Western Cape (UWC), Cape Town, South Africa the students are exposed to research in various modules where they are trained in epidemiology, research methods and literature appraisal. In addition, they personally conduct a research project, performing relevant statistical analyses. The relevant modules for these exposures are offered in the 3rd and final (4th) year of study. Five of the 11 modules in the physiotherapy course in the 3rd and 4th years expose the students to research and evidence-based principles.

However, in this context and other current approaches to teaching these research skills the focus is primarily on gaining skills by participation in the research process, as part of which students must find and appraise evidence from research. ${ }^{[5]}$ This highlights that teaching research methodology is inclined towards equipping students with the skills to conduct research rather than use research for evidence-based practice. ${ }^{[6]}$

It is therefore currently unclear whether students are able to apply the principles of evidence-based practice to patient care. The study objectives were to determine the self-assessed literature-searching behaviour, self-perceived knowledge of critical appraisal skills and beliefs relating to evidence-based practice in final-year undergraduate physiotherapy students.

\section{Methods \\ Design, setting and participants}

This study used an embedded mixed methods design, with a small qualitative component playing a supportive secondary role to the primary quantitative design. The study took place at a local physiotherapy department at UWC, which served as a clinical rotation for final-year physiotherapy students. A convenience sample of all final-year undergraduate physiotherapy students $(N=36)$ registered for the 2012 academic year was approached to participate in the study.

\section{Data collection}

Data were collected using an existing questionnaire, the 'Critical appraisal of medical literature and evidence-based medicine: Participants' knowledge and needs assessment detailed training needs analysis form. ${ }^{\left[{ }^{3}\right]}$ The original questionnaire was developed to measure the basic knowledge of, skills in and beliefs about the main principles of evidence-based practice among allied healthcare professionals and complementary and alternative medicine healthcare practitioners. The questions were related to the participants 
self-assessment of their literature-searching behaviour and self-perceived knowledge of their own critical appraisal skills and beliefs. Responses were captured by multiple-choice answers and 6-point Likert scales, and participants could indicate if they did not understand the question. The questionnaire also included questions about the participants' confidence in assessing research methodology and items relating to their beliefs about evidence-based practice. ${ }^{[7]}$ Content validity of the questionnaire was achieved by asking experts $(n=5)$ who have published in and taught evidence-based practice to complete the questionnaire. Their feedback suggested the removal of all questions relating to qualified practitioners, as the questionnaire was to be completed by undergraduate students. Their second recommendation was that reference to 'evidence-based medicine' should be changed to 'evidence-based practice'. Finally, two open-ended questions regarding the students' rationale behind their treatment choices and their understanding of evidence-based practice replaced a single question on the participants' understanding of evidence-based medicine.

\section{Data collection procedure}

An information sheet describing the study in detail and a consent form ensuring the students' anonymity accompanied the questionnaire. The students were advised that they could withdraw from the study at any time. The questionnaires were distributed by the researcher at the end of a class in the physiotherapy department. The students were asked to complete them and hand them back to the researcher. The study received ethical clearance from UWC, project number 12/3/12. Permission to conduct the survey was obtained from the head of the Department of Physiotherapy.

\section{Data analysis}

The quantitative data obtained from the questionnaire were captured using a Microsoft Excel (USA) sheet and exported for analysis with Statistical Product and Service Solutions version 21 (SPSS 21, IBM Corp, USA). Descriptive statistics were employed to analyse the data, which were presented in frequencies and percentages. The two open-ended questions were analysed thematically. Firstly, emerging and common codes were identified. Categories were developed from the codes and themes emerged from the common categories. An outside reviewer was approached by the researcher to check and validate the themes.

\section{Results}

\section{Exposure to research methodology and access to literature}

A total of 27 students completed the questionnaire, yielding a response rate of $75 \%$. The questionnaire initially explored the exposure of the participants to research and evidence-based practice principles. All the participants were exposed to epidemiology, research methods and literature appraisal. To implement evidence-based practice, participants needed to have access to literature, as it is an important component of evidence-based practice. Therefore, determining whether students had access was important. In this study, all the participants had access to the internet (100\%) and $85 \%$ indicated that they had access to a medical library. Of the participants, $92 \%$ indicated that they had access to the internet on their own computers and $42 \%$ accessed the internet through university resources.

\section{Literature-searching behaviour}

Examining evidence in clinical practice is a skill that students need to implement evidence-based practice. This skill depends on how often students collect evidence, how often they read the literature and the type of material they use to find the evidence they need for their practice. The questionnaire established this by asking how often the students searched for evidence. Thirty percent $(n=8)$ indicated that they searched for literature more than once a week, $52 \%(n=4)$ only read literature for specific information, compared with $7 \%(n=2)$ who stated that they read literature every week, and $85 \%(n=23)$ retrieved information from journal articles to inform their practice.

\section{Computer skills}

Computer skills are necessary for the evidence-based practice process. Results showed that $67 \%$ of participants had written an article using Microsoft Word, 93\% had sent and received emails, 96\% had used the internet to search for information, $89 \%$ had used the internet via a browser and $85 \%$ used databases such as MEDLINE and EBSCOhost.

\section{Confidence and beliefs}

The respondents' confidence in six skills was assessed. The results showed that $48 \%(n=13)$ felt they were slightly confident in assessing the study design and in assessing generalisability of research articles, $44 \%(n=12)$ stated they were slightly confident in evaluating statistical tests and principles and assessing the general worth of the article, $41 \%(n=11)$ indicated they were slightly confident in evaluating bias and $37 \%(n=10)$ were confident in assessing the adequacy of the sample size. Four percent $(n=1)$ stated they had no confidence in evaluating bias or statistical tests and principles.

The students' beliefs in evidence-based practice showed that $33 \%(n=9)$ both slightly disagreed and disagreed that original articles are confusing, $59 \%(n=16)$ strongly agreed that evidence-based practice is essential in their practice, and $37 \%(n=10)$ agreed and $22 \%(n=6)$ strongly agreed (a total of $16 / 27,59 \%$ ) that they feel they needed more training in evidence-based practice. With regard to confidence in assessing research evidence, 59\% $(n=16)$ slightly agreed that they were confident, $44 \%(n=12)$ slightly agreed, $33 \%(n=9)$ agreed and $4 \%(n=1)$ strongly agreed that systematic reviews are key to informing evidence-based practice, 52\% $(n=14)$ disagreed that evidence-based practice has little impact on an individual's practice, $48 \%$ $(n=13)$ only slightly agreed that they received good training in evidencebased practice, $44 \%(n=12)$ slightly disagreed that clinical judgement is more important than evidence-based practice, $37 \%(n=10)$ slightly disagreed that patient choices should override evidence-based practice, and $44 \%(n=12)$ slightly disagreed that evidence-based practice is a passing fashion.

\section{Qualitative data}

There were two open-ended questions about the students' perceptions of evidence-based practice. The first question aimed to explore what the students based their treatment choices on. It was evident from the information that students used literature, role-models, previous experience and patient preferences as evidence on which to base their treatment choices:

'I read articles which support certain treatments.'

'... knowledge gained through clinical supervision sessions.'

'I use my knowledge of what has previously worked in practice.'

'... my findings during the assessments and patient's preferences influence my choices.'

'Knowledge gained through clinical supervision, applied physiotherapy lecture notes, textbooks. 
The second question was about students' understanding of the concept of evidence-based practice. The main idea that emerged was that evidencebased practice was 'treatment that was based on information from journal articles' and 'treatment based on studies that show the effectiveness of a treatment technique.' Quotes to support this include:

'Evidence-based practice is when you constantly look for current journal articles to back up why you have used a specific treatment for a patient and to use journal articles for research when you are unsure about a condition or treatment.'

'Evidence-based practice is techniques that have been practised during a study and have been proven effective.'

\section{Discussion}

The objective of the study was to determine the self-assessment literaturesearching behaviour, self-perceived knowledge of critical appraisal skills and evidence-based practice beliefs among final-year undergraduate physiotherapy students at a local university in SA. Although only a small number participated in the study, the information provides valuable data for future larger studies.

The UWC physiotherapy programme, which was the setting for this study, teaches the principles of evidence-based practice in the form of research methodology lectures. This is similar to other physiotherapy programmes in SA and Norway. ${ }^{[3,6]}$ One study found that although research methods and statistics had been essential components of a Bachelor's programme at a Norwegian university for a number of years, students continued to struggle with finding and using relevant and valid information such as research evidence. ${ }^{[3]}$ The students received little, if any, practical guidance in clinical settings on how to apply research evidence to real patient management. In the same study, ${ }^{[3]}$ the physiotherapy students did attempt evidence-based practice in the clinical setting. In our study, the students were only exposed to the basic skills of research in the 3rd and 4th years of study; the literature states that research skills and the skills of searching, appraising and applying research evidence to individual patients should be taught early and applied as an essential part of learning during the entire curriculum. ${ }^{[8]}$

The majority of the study population indicated that they had access to computers as well as medical literature at university. This was the same for another SA study that also found students had access to the internet on campus. ${ }^{[9]}$

Contrary to the literature ${ }^{[3]}$ that indicated that undergraduate students found it difficult to determine if research evidence was valid and applicable, the students in the current study showed some confidence in assessing research evidence. However, the insecurity of students transitioning to qualified practitioners who will engage in evidence-based practice was evident in their statements that they only felt slightly confident in literature appraisal and wanted more training in evidence-based practice. These findings were similar to those of a study of occupational therapists, which showed that students displayed less confidence when engaging with the processes of evidence-based practice. ${ }^{[10]}$ This was also similar to the study in Norway where it was evident that although students value evidencebased practice and recognise it as a vital part of clinical practice, they continue to feel that they need more training in it. ${ }^{[3]}$ Therefore, the need for more training in applying evidence-based practice is evident across disciplines.

With regard to patients' beliefs and evidence-based practice, the results showed that the majority of the students were in slight disagreement that the patients' preferences should override evidence-based practice. This differed to what was found in the literature. In an Australian study of occupational therapists, the majority agreed that evidence-based practice should be client centred, even though a third of the sample was uncertain, ${ }^{[10]}$ and research conducted in the USA highlighted that evidence-based practice should be patient centred at all times. ${ }^{[11]} \mathrm{A}$ large number of students felt that systematic reviews informed evidence-based practice. Similarly, randomised controlled trials, especially the systematic review of several randomised trials, are very likely to inform rather than mislead us and have become the 'gold standard' for judging whether a treatment does more good than harm. ${ }^{[1]}$ Thus, the need to create an understanding that evidence-based practice is not in lieu of patientcentred practice is essential. Evidence-based practice is the integration of clinical expertise, patient values and the best research evidence into the decision-making process for patient care. Clinical expertise refers to the clinician's accumulated experience, education and clinical skills. The patient brings to the encounter his or her own personal preferences and unique concerns, expectations and values. The best research evidence is usually found in clinically relevant research that has been conducted using sound methodology. ${ }^{[12]}$ Considering this, the understanding of the concept of evidence-based practice in this study seems quite limited. The students based their treatment choices on articles, clinical supervision, knowledge of previous patient encounters, textbooks and what was taught in class. This was similar to findings from a survey that indicated that treatment choices were largely based on initial training and, to a lesser degree, on journal research articles. ${ }^{[13]}$

\section{Conclusion}

The results of the questionnaire highlighted that more than half the sample searched for literature for specific information and the majority used journal articles to inform their practice. The majority also had access to computers and literature. Less than half were, however, only slightly confident in appraising literature. They believed that evidence-based practice was important but felt they need more training. The students' understanding of the concept of evidence-based practice was limited compared with the literature. ${ }^{[12]}$ Some based their treatment choices on content, clinical supervisors, what was taught in class and others' journal articles. Some believed evidence-based practice was the practice of basing treatment choices on sound clinical evidence and others believed it to be techniques found to be effective in a study.

\section{Clinical relevance of findings}

The findings suggest that the final-year undergraduate students have an awareness of the concept of evidence-based practice; however, they do not seem to be implementing it completely in clinical practice. Therefore, it may be worthwhile teaching the principles of evidence-based practice in the undergraduate clinical practice module and not just the research methodology module. Other research has suggested that the incorporation of all five steps from the Sicily model in clinical practice should be investigated. ${ }^{[6]}$

\section{Study limitations}

The study had a small sample size and only final-year students from one university were approached to participate; however, the findings provide a basis from which health professions educators may operate when teaching evidence-based practice. 


\section{Suggestions for further research}

It may be valuable to explore the teaching strategies used for the implementation of evidence-based practice into physiotherapy education and whether the evidence-based practice process should be taught in the clinical setting.

\section{References}

1. Sackett DL, Rosenberg WM, Gray JA, Haynes RB, Richardson WS. Evidence based medicine: What it is and what it isn't. BMJ 1996;312(7023):71-72. DOI:10.1136/bmj.312.7023.71

2. Long K, McEvoy M, Lewis L, Wiles L, Williams M, Olds T. Entry-level evidenced-based practice training in physiotherapy students: Does it change knowledge, attitudes, and behaviours? A longitudinal study. Internet Allied Health Sci Pract 2011;9(3)

3. Olsen NR, Bradley P, Lomborg K, Nortvedt MW. Evidence-based practice in clinical physiotherapy education: A qualitative interpretive description. BMC Med Educ 2013;13(1):52. DOI:10.1186/1472-6920-13-52

4. Dawes M, Summerskill W, Glasziou P, et al. Sicily statement on evidence-based practice. BMC Med Educ 2005;5(1):1. DOI:10.1186/1472-6920-5-1
5. French B. Developing the skills required for evidence-based practice. Nurse Educ Today $1998 ; 18(1): 46-51$

6. Burger $\mathrm{M}$, Louw $\mathrm{Q}$. Integrating evidence-based principles into the undergraduate physiotherapy research methodology curriculum: Reflections on a new approach. Afr I Health Professions Educ 2014:6(2):198-202. DOI:10.7196/AJHPE516

7. Hadley J, Hassan I, Khan K. Knowledge and beliefs concerning evidence-based practice amongst complementary and alternative medicine health care practitioners and allied health care professionals: A questionnaire survey. BMC Complement Altern Med 2008;8(1):45. DOI:10.1186/1472-6882-8-45

Glasziou P, Burls A, Gilbert, R. Evidence based medicine and the medical curriculum. BMJ 2008;337(3):704-705. DOI:10.1136/bmj.al253

9. Rowe M, Frantz J, Bozalek V. Physiotherapy student's use of online technologies as part of their learning practices: A case study. S Afr J Physiother 2012;68(1):29-34.

10. Bennett S, Tooth L Mc Kenna K, et al. Perceptions of evidence-based practice: A survey of Australian occupational therapists. Aust Occup Ther J 2003;50(1):13-22. DOI:10.1046/j.1440-1630.2003.00341.x

1. Manske R, Lehecka B. Evidence-based medicine/practice in sports physical therapy. Int J Sports Phys Ther 2012;7(5):461-473.

12. Sackett D. Evidence-based practice. 2002. http://mcgrawhill.co.uk/openup/chapters/9780335244737.pd (accessed 30 December 2015)

13. Turner A, Whitfield A. Physiotherapists' reasons for selection of treatment techniques: A cross-national survey. Physiother Theory Pract 1999;15(4):235-246. DOI:10.1080/095939899307649 